\title{
Transitive Closure of Interval-valued Fuzzy Relations
}

\author{
Ramón González-del-Campo ${ }^{1}$, Luis Garmendia ${ }^{2}$, Jordi Recasens ${ }^{3}$ \\ ${ }^{1}$ DSIC, Universidad Complutense de Madrid, Spain \\ E-mail: rgonzale@estad.ucm.es \\ 2 DISIA, Universidad Complutense de Madrid, Spain \\ E-mail: lgarmend@fdi.ucm.es \\ ${ }^{3}$ Universitat Politecnica de Catalunya, Spain \\ E-mail: j.recasens@upc.edu \\ Received 16 December 2011 \\ Accepted 14 December 2012
}

\begin{abstract}
In this paper are introduced some concepts of interval-valued fuzzy relations and some of their properties: reflexivity, symmetry, T-transitivity, composition and local reflexivity. The existence and uniqueness of T-transitive closure of interval-valued fuzzy relations is proved. An algorithm to compute the T-transitive closure of finite interval-valued fuzzy relations is showed. Some properties and some examples is given for t-representable and t-pseudo representable generalized t-norms.
\end{abstract}

Keywords: Generalized t-norms, Interval-valued Fuzzy Relations, Interval-valued Fuzzy Sets, t-norms, t-representable t-norms, T-transitive closure.

\section{Introduction}

Fuzzy sets, $\mathscr{F} \mathscr{S}$, were introduced by Zadeh in $1.965^{20}$. Since then many generalizations of fuzzy sets have been proposed to model the uncertainty and the vagueness in linguistic variables replacing the unit interval by another structure such as posets or lattices $5,15,13$. One of these generalizations are type-2 fuzzy sets, $\mathscr{F} \mathscr{S} 2,{ }^{21,22,23}$ were introduced by Zadeh. A Type-2 fuzzy set $A$ on a universe of discourse $X, \mathscr{F} \mathscr{S} 2$, is a fuzzy set whose membership function is another fuzzy set on $[0,1]$ :

$$
A=\left\{\left((x, u), \mu_{A}(x, u)\right) \mid \forall x \in X, \forall u \in[0,1]\right\}
$$

Type-2 fuzzy sets have been widely studied and applied since in many cases the uncertainty can be better expressed by a fuzzy set than by a single numeric value. The problem with type 2 fuzzy sets though is their computational complexity and the difficulty for an expert to select the adequate fuzzy subset as membership degree of an object to a linguistic label. This is why some simplifications have been proposed, such as the use of only some families of fuzzy sets such as triangular and trapezoidal ones.

Interval-valued fuzzy sets $(\mathscr{I} \mathscr{V} \mathscr{F} \mathscr{S})$ were introduced in the 60 s by Grattan-Guinness ${ }^{14}$, Jahn ${ }^{16}$, Sambuc ${ }^{19}$ and Zadeh ${ }^{21}$. They are extensions of classical fuzzy sets where the membership value between 0 and 1 is replaced by an interval in $[0,1]$. They easily allow to model uncertainty and vagueness because sometimes it is easier for experts to give a "membership interval" than a membership degree to objects on a universe. $\mathscr{I} \mathscr{V} \mathscr{F} \mathscr{S}$ are a special case of type-2 fuzzy sets that simplifies the calculations while preserving their richness as well. The intuitionistic fuzzy sets on $X(\mathscr{I} \mathscr{F} \mathscr{S})$ intro- 
duced by Atanassov ${ }^{1}$ are also a extension of fuzzy sets in which each element has a membership degree, $\mu$, and a non-membership degree, $v$ satisfying: $\mu+v \leqslant 1$.

$$
A=\{(x, \mu(x), v(x)) \mid x \in X, \mu(x), v(x) \in[0,1]\}
$$

The value $\pi=1-\mu-v$ is a measure of the uncertainty. Intuitionistic relations are been studied widely $3,4,9,12,11,18$.

This paper is organized as follows: In section three the $\mathscr{T}$-transitive closure of interval-valued fuzzy relations is defined and it is showed that it always exists and it is unique. In section four $\mathscr{T}$ transitive closure of interval-valued fuzzy relations is studied. Traditionally, the study of conjunctions between interval-valued fuzzy sets has been reduced to be modeled with t-representable t-norms. However, not all generalized t-norms are t-representable. Moreover, some of the non t-representable t-norms sometimes satisfy even more properties than $t$ representable t-norms ${ }^{6}$. Probably the most important property a fuzzy relation can fulfil is transitivity with respect a given t-norm. Since many times the data are given by a proximity relation $\mathrm{P}$ (i.e.: a reflexive and symmetric but not necessarily transitive fuzzy relation), there are some methods to obtain a transitive relation close to $P$ to replace it when transitivity is required. The most popular way to do this is calculating its transitive closure. In section four we introduce the concept of T-transitive closure for an interval-valued fuzzy relation and its expression in a finite universe for any generalized t-norm. A few methods to compute it and some examples are given. In section five an algorithm to compute the $\mathscr{T}$-transitive closure is given and some examples shown. In section six a very simple application is shown.

\section{Preliminaries}

Definition $2.1{ }^{7}$ Let $L$ be the lattice of intervals in [0,1] that satisfies:

1. $L=\left\{\left[x_{1}, x_{2}\right] \in[0,1]^{2}\right.$ with $\left.x_{1} \leqslant x_{2}\right\}$.

2. $\left[x_{1}, x_{2}\right] \leqslant L\left[y_{1}, y_{2}\right]$ if and only if $x_{1} \leqslant y_{1}$ and $x_{2} \leqslant y_{2}$

Also by definition:

$$
\begin{aligned}
& {\left[x_{1}, x_{2}\right]<_{L}\left[y_{1}, y_{2}\right] \Leftrightarrow x_{1}<y_{1}, x_{2} \leqslant y_{2} \text { or }} \\
& x_{1} \leqslant y_{1}, x_{2}<y_{2} \\
& {\left[x_{1}, x_{2}\right]={ }_{L}\left[y_{1}, y_{2}\right] \Leftrightarrow x_{1}=y_{1}, x_{2}=y_{2} .}
\end{aligned}
$$

$0_{L}={ }_{L}[0,0]$ and $1_{L}={ }_{L}[1,1]$ are the smallest and the greatest elements in $L$ respectively.

$L$ is a complete lattice and the supremum and infimum are defined as follows.

Definition 2.2 ${ }^{6}$ Let $\left\{\left[v_{i}, w_{i}\right]\right\}$ be a set of intervals on L. Then

$$
\begin{aligned}
& \text { 1. } \wedge\left\{\left[v_{i}, w_{i}\right]\right\} \equiv\left[\inf \left\{v_{i}\right\}, \inf \left\{w_{i}\right\}\right] \\
& \text { 2. } \vee\left\{\left[v_{i}, w_{i}\right]\right\} \equiv\left[\sup \left\{v_{i}\right\}, \sup \left\{w_{i}\right\}\right]
\end{aligned}
$$

Definition 2.3 ${ }^{7}$ An interval-valued fuzzy set $A$ on a universe $X$ can be represented by the mapping:

$$
A=\left\{\left(a,\left[x_{1}, x_{2}\right]\right) \mid a \in X,\left[x_{1}, x_{2}\right] \in L\right\}
$$

Definition $2.4^{7}$ Let $X$ be a universe and $A$ and $B$ two interval-valued fuzzy sets. The equality between $A$ and $B$ is defined as: $A={ }_{L} B$ if and only if $A(a)={ }_{L} B(a) \forall a \in X$.

Definition $2.5{ }^{7}$ Let $X$ be a universe and $A$ and $B$ two interval-valued fuzzy sets. The inclusion of $A$ in to $B$ is defined as: $A \subseteq_{L} B$ if and only if $A(a) \leqslant_{L} B(a)$ $\forall a \in X$.

Definition 2.6 ${ }^{7}$ A negation function for intervalvalued fuzzy sets $\mathscr{N}$ is a decreasing function, $\mathscr{N}$ : $L \rightarrow L$, that satisfies:

$$
\begin{aligned}
& \text { 1. } \mathscr{N}\left(0_{L}\right)={ }_{L} 1_{L} \\
& \text { 2. } \mathscr{N}\left(1_{L}\right)={ }_{L} 0_{L}
\end{aligned}
$$

If $\mathscr{N}\left(\mathscr{N}\left(\left[x_{1}, x_{2}\right]\right)\right)={ }_{L}\left[x_{1}, x_{2}\right]$ for all $\left[x_{1}, x_{2}\right]$ in $L$ then $\mathscr{N}$ is called an involutive negation.

Definition 2.7 A strong negation function for interval-valued fuzzy sets, $\mathscr{N}$, is a involutive function, $\mathscr{N}: L \rightarrow L$, that satisfies:

$$
\begin{aligned}
& \text { 1. } \mathscr{N}\left(0_{L}\right)={ }_{L} 1_{L} \\
& \text { 2. } \mathscr{N}\left(1_{L}\right)={ }_{L} 0_{L}
\end{aligned}
$$

Example 2.1 Let $\mathscr{N}$ be the involutive mapping defined by:

$$
\begin{aligned}
& \mathscr{N}: L \rightarrow L \\
& \mathscr{N}\left(\left[x_{1}, x_{2}\right]\right)={ }_{L}\left[1-x_{2}, 1-x_{1}\right]
\end{aligned}
$$


Then $\mathscr{N}$ is a negation operator for interval-valued fuzzy sets. It is trivial to prove that: $\mathscr{N}\left(0_{L}\right)={ }_{L} 1_{L}$, $\mathscr{N}\left(1_{L}\right)={ }_{L} 0_{L}$ and $\mathscr{N}\left(\mathscr{N}\left(\left[x_{1}, x_{2}\right]\right)\right)={ }_{L}\left[x_{1}, x_{2}\right]$.

T-norms are generalized to the lattice $L$ in a straightforward way.

Definition $2.8{ }^{7}$ A t-norm on L function $\mathscr{T}$ is a monotone increasing, symmetric and associative operator, $\mathscr{T}: L^{2} \rightarrow L$, that satisfies: $\mathscr{T}\left(1_{L},\left[x_{1}, x_{2}\right]\right)={ }_{L}$ $\left[x_{1}, x_{2}\right]$ for all $\left[x_{1}, x_{2}\right]$ in $L$.

Due to monotony, it is easy to show:

$$
\begin{gathered}
\mathscr{T}\left(\text { Joint }\left\{\left[v_{i}, w_{i}\right]\right\},\left[y_{1}, y_{2}\right]\right) \geqslant_{L} \\
\text { Joint }\left\{\mathscr{T}\left(\left[v_{i}, w_{i}\right],\left[y_{1}, y_{2}\right]\right)\right\} \\
\mathscr{T}\left(\text { Meet }\left\{\left[v_{i}, w_{i}\right]\right\},\left[y_{1}, y_{2}\right]\right) \leqslant L \\
\text { Meet }\left\{\mathscr{T}\left(\left[v_{i}, w_{i}\right],\left[y_{1}, y_{2}\right]\right)\right\}
\end{gathered}
$$

Due to the associativity of $\mathscr{T}$ the conjunction of three or more intervals can be defined inductively as:

$$
\begin{gathered}
\mathscr{T}(a, \mathscr{T}(b, c))={ }_{L} \mathscr{T}(\mathscr{T}(a, b), c)={ }_{L} a \triangle b \triangle c \\
\text { where } \triangle=_{L} \mathscr{T} .
\end{gathered}
$$

where $a={ }_{L}\left[a_{1}, a_{2}\right], b={ }_{L}\left[b_{1}, b_{2}\right]$ and $c={ }_{L}\left[c_{1}, c_{2}\right]$.

Example 2.2 Let In $f_{L}$ be defined as follows:

$$
\operatorname{Inf}_{L}\left(\left[x_{1}, x_{2}\right],\left[y_{1}, y_{2}\right]\right)=\operatorname{Meet}\left\{\left[x_{1}, x_{2}\right],\left[y_{1}, y_{2}\right]\right\}
$$

It easy to prove that In $f_{L}$ is a t-norm on $L$.

$\underline{T}\left(\left[x_{1}, x_{2}\right],\left[y_{1}, y_{2}\right]\right) \quad$ and $\bar{T}\left(\left[x_{1}, x_{2}\right],\left[y_{1}, y_{2}\right]\right)$ will denote the lower and the higher values of $T\left(\left[x_{1}, x_{2}\right],\left[y_{1}, y_{2}\right]\right)$.

Definition $2.9^{2}$ Let $\left\{x_{i}\right\}$ in $[0,1]$. A t-norm $T$ in $([0,1], \leqslant)$ is left-continuous if it satisfies:

$$
T\left(\operatorname{Sup} x_{i}, y\right)=\operatorname{Sup} T\left(x_{i}, y\right)
$$

Right-continuity can be defined in a similar way. This property is also called sup-preserving.

Definition 2.10 ${ }^{7}$ A t-norm on L operator $\mathscr{T}$ is $t$ representable in $L$ if there are two t-norms: $T_{1}$ and $T_{2}\left(T_{1}, T_{2}\right.$, in $\left.([0,1], \leqslant)\right)$ that satisfy:

$$
\mathscr{T}\left(\left[x_{1}, x_{2}\right],\left[y_{1}, y_{2}\right]\right)={ }_{L}\left[T_{1}\left(x_{1}, y_{1}\right), T_{2}\left(x_{2}, y_{2}\right)\right]
$$

where $T_{1}(v, w) \leqslant T_{2}(v, w) \forall v, w \in[0,1]$.

Let $x={ }_{L}\left[x_{1}, x_{2}\right]$ and $y={ }_{L}\left[y_{1}, y_{2}\right]$ be two intervals on L:

Example 2.3 $\mathscr{T}={ }_{L}\left[\min \left(x_{1}, y_{1}\right), \min \left(x_{2}, y_{2}\right)\right]$ is $t$ representable in $([0,1], \leqslant)$. Note that $\min$ is the highest $t$-norm.

Example 2.4 The following product t-norm on $L \mathscr{T}$ is t-representable:

$$
\mathscr{T}\left(\left[x_{1}, x_{2}\right],\left[y_{1}, y_{2}\right]\right)={ }_{L}\left[x_{1} * y_{1}, x_{2} * y_{2}\right]
$$

Example 2.5 There are two generalizations of the Lukasiewicz t-norm ${ }^{6}$ :

- $T_{w}\left(\left[x_{1}, x_{2}\right],\left[y_{1}, y_{2}\right]\right)=_{L}$ $\left[\max \left(0, x_{1}+y_{1}-1\right), \max \left(0, x_{2}+y_{2}-1\right)\right]$

- $T_{W}\left(\left[x_{1}, x_{2}\right],\left[y_{1}, y_{2}\right]\right)={ }_{L}$ $\left[\max \left(0, x_{1}+y_{1}-1\right), \max \left(0, x_{1}+y_{2}-1, x_{2}+y_{1}-\right.\right.$ 1)]

Note that $T_{w}$ is t-representable but $T_{W}$ is not.

Definition 2.11 ${ }^{10}$ A t-norm on L operator $\mathscr{T}$ is pseudo-t-representable in $\mathscr{L}^{I}$ if there exists a t-norm $T$ in $([0,1], \leqslant)$ that satisfies:

$$
\begin{gathered}
\mathscr{T}\left(\left[x_{1}, x_{2}\right],\left[y_{1}, y_{2}\right]\right)={ }_{L} \\
{\left[T\left(x_{1}, y_{1}\right), \max \left\{T\left(x_{1}, y_{2}\right), T\left(x_{2}, y_{1}\right)\right\}\right]}
\end{gathered}
$$

The t-norm $T$ is called the representant of $\mathscr{T}$.

Example 2.6 It is showed some examples of pseudo-t-representable t-norms on $L$ :

\begin{tabular}{|c|c|}
\hline$T$ & $\mathscr{T}$ \\
\hline \hline $\min (x, y)$ & {$\left[\min \left(x_{1}, y_{1}\right), \max \left(\min \left(x_{1}, y_{2}\right), \min \left(x_{2}, y_{1}\right)\right)\right]$} \\
\hline$x * y$ & {$\left[x_{1} * y_{1}, \max \left(x_{1} * y_{2}, x_{2} * y_{1}\right)\right]$} \\
\hline $\max (0, x+y-1)$ & {$\left[\max \left(0, x_{1}+y_{1}-1\right), \max \left(0, x_{1}+y_{2}-1, x_{2}+y_{1}-1\right)\right]$} \\
\hline
\end{tabular}

Definition $2.12{ }^{7}$ A t-conorm on $L \mathscr{S}$ is an increasing, commutative, and associative operator $\mathscr{S}$ : $L^{2} \rightarrow L$, that satisfies: $\mathscr{S}\left(0_{L},\left[x_{1}, x_{2}\right]\right)={ }_{L}\left[x_{1}, x_{2}\right]$.

Due to the associativity of $\mathscr{S}$ we can write:

$$
\begin{gathered}
\mathscr{S}(a, \mathscr{S}(b, c))={ }_{L} \mathscr{S}(\mathscr{S}(a, b), c)={ }_{L} a \nabla b \nabla c \\
\text { where } \nabla={ }_{L} \mathscr{S} .
\end{gathered}
$$

Example 2.7 Let Sup $_{L}$ be defined as follows:

$$
\operatorname{Sup}_{L}\left(\left[x_{1}, x_{2}\right],\left[y_{1}, y_{2}\right]\right)=\operatorname{Joint}\left\{\left[x_{1}, x_{2}\right],\left[y_{1}, y_{2}\right]\right\}
$$

It easy to prove that $\mathrm{Sup}_{L}$ is a t-conorm on $L$.

In this paper the following definitions are proposed.

Definition 2.13 Let $\left\{\left[v_{i}, w_{i}\right]\right\}$ be in L. A t-norm on $L$ operator $\mathscr{T}$ is sup-preserving if and only if:

$$
\begin{gathered}
\mathscr{T}\left(\operatorname{Sup}_{L}\left\{\left[v_{i}, w_{i}\right]\right\},\left[y_{1}, y_{2}\right]\right)={ }_{L} \\
\operatorname{Sup}_{L}\left\{\mathscr{T}\left(\left[v_{i}, w_{i}\right],\left[y_{1}, y_{2}\right]\right)\right\}
\end{gathered}
$$

Definition 2.14 Given a t-norm on $L \mathscr{T}$ and a generalized negation $\mathscr{N}$, the operator:

$$
\mathscr{T}_{\mathscr{N}}^{*}={ }_{L} \mathscr{N}\left(\mathscr{T}\left(\mathscr{N}\left(\left[x_{1}, x_{2}\right]\right), \mathscr{N}\left(\left[y_{1}, y_{2}\right]\right)\right)\right.
$$

is a t-conorm on $L$ called dual t-conorm of $\mathscr{T}$ with respect to $\mathscr{N}$.

A t-norm, a negation and the dual t-conorm of $\mathscr{T}$ with respect to $\mathscr{N}$ is called a De Morgan triplet. 


\section{On the existence of $\mathscr{T}$-transitive closure of interval-valued fuzzy relations}

Definition 3.1 An interval-valued fuzzy relation $R$ : $X^{2} \rightarrow L$ is a generalized $\mathscr{T}$-indistinguishability if it is reflexive, symmetric and $\mathscr{T}$-transitive.

Definition 3.2 Let $L$ be the lattice of intervals in $[0,1]$ and let $P$ be a property. Let $R: X^{2} \rightarrow L$ be a relation on a finite universe $X$. The $P$ closure of $R$ is the relation $R^{\mathscr{P}}: X \times X \rightarrow L$ that satisfies:

1. $R^{\mathscr{P}}$ satisfies $P$.

2. $R \subseteq_{L} R^{\mathscr{P}}$.

3. If $R \subseteq_{L} R^{\prime}$ and $R^{\prime}$ satisfies $P$ then $R^{\mathscr{P}} \subseteq_{L} R^{\prime}$.

Lemma 3.1 Let $R$ be an interval-valued fuzzy relation in a universe $X$ and let $\mathscr{T}$ be an arbitrary $t$ norm on $L$. Then the $\mathscr{T}$-transitive closure of $R$ always exists.

Proof. In similar way than ${ }^{2}$ let Meet the greatest lower bound of any subset of elements on $L$. Consider the set $\Omega_{R}$ of $\mathscr{T}$-transitive fuzzy relations containing $R$. Let us define the fuzzy relation $S_{R}^{*}(a, b)={ }_{L}$ Meet $_{S \in \Omega_{R}}\{S(a, b)\}$. We will prove that $S_{R}^{*}$ is $\mathscr{T}$-transitive. Due to monotony of generalized $\mathscr{T}$-norms it is obtained:

$\mathscr{T}\left(S_{R}^{*}(a, b), S_{R}^{*}(b, c)\right)=$

$\mathscr{T}\left(\right.$ Meet $_{S_{1} \in \Omega_{R}}\left\{S_{1}(a, b)\right\}$, Meet $\left._{S_{2} \in \Omega_{R}}\left\{S_{2}(b, c)\right\}\right)$

$\leqslant$ Meet $_{S_{1} \in \Omega_{R}}$ Meet $_{S_{2} \in \Omega_{R}}\left\{\mathscr{T}\left(S_{1}(a, b), S_{2}(b, c)\right)\right\}$

$\leqslant \operatorname{Meet}_{S \in \Omega_{R}}\{\mathscr{T}(S(a, b), S(b, c))\}=S_{R}^{*}(a, c) \square$

Lemma 3.2 If $R$ be an interval-valued relation then $\mathscr{T}$-transitive closure is unique.

Proof. Let $S_{1}$ and $S_{2}$ be two relations. If $S_{1}$ and $S_{2}$ are transitive closures of $R$ then according to definition 3.2: $S_{1} \subseteq_{L} S_{2}$ and $S_{2} \subseteq_{L} S_{1}$, consequently $S_{1}={ }_{L} S_{2}$

\section{4. $\mathscr{T}$-transitive closure of interval-valued fuzzy relations}

The calculation of $\mathscr{T}$-transitive closure of intervalvalued fuzzy relations via sup- $\mathscr{T}$ is showed. The sup - $\mathscr{T}$ product is associative if $\mathscr{T}$ is suppreserving and the universe is finite. Moreover, it is proved that the sup $-\mathscr{T}$ product is continuous and so is the calculation of the $\mathscr{T}$-transitive closure for finite universes. An algorithm to calculate the $\mathscr{T}$ transitive closure is provided.

Proposition 4.1 If $\mathscr{T}$ is t-representable with $T_{1}$ and $T_{2}\left(\mathscr{T}=\left[T_{1}, T_{2}\right]\right)$ then an interval-valued relation $R: X^{2} \rightarrow L$ is $\mathscr{T}$-transitive if and only if $\underline{R}$ is $T_{1}$ transitive and $\bar{R}$ is $T_{2}$-transitive.

Definition 4.1 Let $\mathscr{T}$ be a t-norm on L. Let $\mathscr{S}$ be a $t$-conorm on $L$ and let $\nabla$ be the $n$-ary $t$-conorm on $L$ defined by associativity. Let $R_{1}$ and $R_{2}$ be two interval-valued fuzzy relations on a finite set $X=\left\{c_{1}, \ldots, c_{m}\right\}$. The $\mathscr{S}-\mathscr{T}$-composition of $R_{1}$ and $R_{2}$ is defined as follows:

$$
\begin{gathered}
\left(R_{1} \diamond(\mathscr{S} \mathscr{T}) R_{2}\right)\left(c_{q}, c_{r}\right)={ }_{L} \\
\nabla_{c_{k} \in X} \mathscr{T}\left(R_{1}\left(c_{q}, c_{k}\right), R_{2}\left(c_{k}, c_{r}\right)\right)
\end{gathered}
$$

where:

$$
\begin{aligned}
& \nabla c_{k} \in X \\
& \quad{ }_{L} \mathscr{T}\left(R_{1}\left(c_{q}, c_{k}\right), R_{2}\left(c_{k}, c_{r}\right)\right)= \\
& \left.\quad \mathscr{T}\left(R_{1}\left(c_{q}, c_{1}\right), R_{2}, c_{m}\right), R_{2}\left(c_{m}, c_{r}\right)\right) \nabla \cdots \nabla
\end{aligned}
$$

Definition 4.2 The local equality relation of a interval-valued fuzzy relation $R$ on $X$ is the fuzzy relation $E_{R}$ is:

$$
\begin{gathered}
E_{R}(a, b)= \\
\begin{cases}\operatorname{Sup}_{L \forall c \in X} \max (R(a, c), R(c, a)) & a=b ; \\
{[0,0],} & a \neq b .\end{cases}
\end{gathered}
$$

Definition 4.3 An interval-valued relation $R$ is locally reflexive if $E_{R} \subseteq_{L} R$.

It is easy to prove that if $R$ is a reflexive intervalvalued relation then $R$ is a locally reflexive intervalvalued relation.

Lemma 4.1 $R$ is $\mathscr{T}$-transitive if and only if $R \diamond_{\text {Sup }_{L} T}$ $R \subseteq_{L} R$.

Proof. The proof is a straightforward generalization of the proof for fuzzy relations.

- $R$ is $\mathscr{T}$-transitive $\Rightarrow R \diamond_{\operatorname{Sup}_{L} T} R \subseteq_{L} R$ :

$\left(R \diamond_{\operatorname{Sup}_{L} T} R\right)(a, b)={ }_{L}$

$\operatorname{Sup}_{L c \in X}\{\mathscr{T}(R(a, c), R(c, b))\} \leqslant{ }_{L} R(a, b)$

due to $\mathscr{T}(R(a, c), R(c, b)) \leqslant{ }_{L} R(a, b) \forall a, b, c \in X$

- $R \diamond_{\operatorname{Sup}_{L} T} R \subseteq_{L} R \Rightarrow R$ is $\mathscr{T}$-transitive:

$\operatorname{Sup}_{L d \in X}\{\mathscr{T}(R(a, d), R(d, b))\} \leqslant_{L} R(a, b) \forall a, b \in$ $X$

Then $\forall c \in X \mathscr{T}(R(a, c), R(c, b)) \leqslant{ }_{L} R(a, b)$ 
Proposition 4.2 Let $X$ be an arbitrary universe. Let $\left\{S_{1}, \ldots, S_{n}\right\}$ a set of interval-valued relations. If $\mathscr{T}$ is a sup-preserving generalized $\mathscr{T}$-norm, then it satisfies:

$$
\begin{aligned}
& R \diamond_{\operatorname{Sup}_{L} \mathscr{T}}\left(\operatorname{Sup}_{L \forall i=1 . . n}\left\{S_{i}\right\}\right)={ }_{L} \\
& \quad \operatorname{Sup}_{L \forall i=1 . . n}\left\{R \diamond_{\text {uup }_{L}} \mathscr{T} S_{i}\right\}
\end{aligned}
$$

Proof. The proof is a straightforward generalization of the proof for fuzzy relations.

$$
\begin{aligned}
& R \diamond_{\operatorname{Sup}_{L} \mathscr{T}}\left(\operatorname{Sup}_{L \forall i=1 . . .}\left\{S_{i}\right\}\right)(a, c)={ }_{L} \\
& \operatorname{Sup}_{L b \in X}\left\{\mathscr{T}\left(R(a, b), \operatorname{Sup}_{L \forall i=1 . . n}\left\{S_{i}(b, c)\right)\right\}\right. \\
& ={ }_{L} \operatorname{Sup}_{L \forall i=1 . . n} \operatorname{Sup}_{L b \in X}\left\{\mathscr{T}\left(R(a, b), S_{i}(b, c)\right)\right\} \\
& ={ }_{L}\left(\operatorname{Sup}_{L \forall i=1 . . . n}\left\{R \diamond_{\operatorname{Sup}_{L}} \mathscr{T} S_{i}\right\}\right)(a, c)
\end{aligned}
$$

Definition 4.4 Given a t-norm on $L \mathscr{T}$, the $\mathscr{T}$ power $R^{(n)_{\mathscr{T}}}$ of a fuzzy relation $R$ on $X$ is recursively defined as follows:

1. $R^{(1) \mathscr{T}} \equiv R$

2. $R^{(n)_{\mathscr{T}}} \equiv R^{(n-1)_{\mathscr{T}}} \diamond_{\text {Sup }_{L} \mathscr{T}} R$

Lemma 4.2 If $A \subseteq_{L} B$ then $A^{(k)} \subseteq_{L} B^{(k)}$

Proof. Trivial due to monotony of $\mathscr{T}$

Lemma $4.3{ }^{8}$ Let $\mathscr{T}$ be any $t$-norm on $L$, then: $\mathscr{T}\left(\left[x_{1}, x_{2}\right],\left[y_{1}, y_{2}\right]\right) \leqslant{ }_{L} \operatorname{Inf}_{L}\left(\left[x_{1}, x_{2}\right],\left[y_{1}, y_{2}\right]\right)$

$$
\forall\left[x_{1}, x_{2}\right],\left[y_{1}, y_{2}\right] \in L
$$

Lemma $4.4{ }^{8}$ Let $\mathscr{S}$ be any $t$-conorm on $L$, then:

$$
\begin{gathered}
\mathscr{S}\left(\left[x_{1}, x_{2}\right],\left[y_{1}, y_{2}\right]\right) \geqslant_{L} \operatorname{Sup}_{L}\left(\left[x_{1}, x_{2}\right],\left[y_{1}, y_{2}\right]\right) \\
\forall\left[x_{1}, x_{2}\right],\left[y_{1}, y_{2}\right] \in L
\end{gathered}
$$

Lemma 4.5 If $\mathscr{T}$ is t-representable with $T_{1}, T_{2}$ in $([0,1], \leqslant)$ then:

$$
R^{(n) \mathscr{T}}=\left[\underline{R}^{(n)_{T_{1}}}, \bar{R}^{(n)_{T_{2}}}\right]
$$

Proof. $R^{(n)_{\mathscr{T}}}\left(c_{q}, c_{r}\right) \equiv R_{q, r}^{(n)_{\mathscr{T}}}=$

$$
\begin{aligned}
& =\operatorname{Sup}_{L k \in X} \mathscr{T}\left(R_{q, k}^{(n-1)_{\mathscr{T}}}, R_{k, r}\right) \\
& =\operatorname{Sup}_{L k \in X}\left[T_{1}\left(\underline{R}_{q, k}^{(n-1)_{\mathscr{T}}}, \underline{R}_{k, r}\right), T_{2}\left(\bar{R}_{q, k}^{(n-1)_{\mathscr{T}}}, \bar{R}_{k, r}\right)\right] \\
& =\left[\max T_{1}\left(\underline{R}_{q, k}^{(n-1)_{\mathscr{T}}}, \underline{R}_{k, r}\right), \max T_{2}\left(\bar{R}_{q, k}^{(n-1)_{\mathscr{T}}}, \bar{R}_{k, r}\right)\right] \\
& =\left[\underline{R}_{q, r}^{(n-1)_{\mathscr{T}}} \circ_{T_{1}} \underline{R}_{q, r}, \bar{R}_{q, r}^{(n-1)_{\mathscr{T}}} \circ_{T_{2}} \bar{R}_{q, r}\right] \\
& =\quad\left[\max _{k \in X} T_{1}\left(\underline{R}_{q, k}^{(n-2)_{\mathscr{T}}}, \underline{R}_{k, r}\right){ }^{\circ} T_{1}\right. \\
& \underline{R}_{q, r}, \max _{k \in X} T_{2}\left(\underline{R}_{q, k}^{(n-2)}, \bar{R}_{k, r} \circ_{T_{2}} \bar{R}_{q, r}\right] \\
& =\left[\left(\underline{R}^{(n-2)_{\mathscr{T}}} \circ_{T_{1}} \underline{R}^{(2)_{T_{1}}}\right)_{q, r},\left(\bar{R}^{(n-2)_{\mathscr{T}} \circ_{T_{2}}}\right.\right.
\end{aligned}
$$

$$
\begin{aligned}
& \left.\left.\bar{R}^{(2)_{T_{2}}}\right)_{q, r}\right] \\
& =\underbrace{\ldots}_{n-2 \text { times }}=\left[\underline{R}^{(n)_{T_{1}}}, \bar{R}^{(n)_{T_{2}}}\right]
\end{aligned}
$$

Theorem 4.1 Let $\mathscr{T}$ be a t-representable t-norm $\left(\mathscr{T}=\left[T_{1}, T_{2}\right]\right)$ and let $R=[\underline{R}, \bar{R}]$ be a interval-valued relation. Then, the $\mathscr{T}$-transitive closure intervalvalued of $R, R^{\mathscr{T}}$, satisfies:

$$
R^{\mathscr{T}}=\left[\underline{R}^{T_{1}}, \bar{R}^{T_{2}}\right]
$$

Proof. Trivial due to lemma 4.5

Lemma 4.6 If $\mathscr{T}$ is pseudo-t-representable with $T$, then:

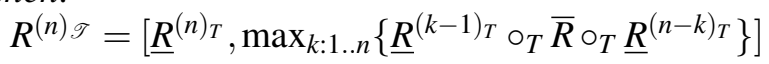

Proof. Note that the composition of fuzzy relations is associative and distributes over "inf" and "sup" fuzzy relations. Note that $R^{(1)_{T}}$ is the relation identity. It is verified to $n=2$. Let's suppose that

$$
R^{(n)_{\mathscr{T}}}=\left[\underline{R}^{(n)_{T}}, \max _{k: 1 . . .}\left\{\underline{R}^{(k-1)_{T}} \circ_{T} \bar{R} \circ_{T} \underline{R}^{(n-k)_{T}}\right\}\right]
$$

Then:

$$
\begin{aligned}
& R^{(n+1)_{\mathscr{T}}}= \\
& =\left[\underline{R}^{(n+1)_{T}}, \max \left\{\bar{R}^{(n)_{\mathscr{T}}} \circ_{T} \underline{R}, \underline{R}^{(n)_{\mathscr{T}}} \circ_{T}\right.\right. \\
& \overline{\bar{R}}\}] \\
& \quad=\left[\underline{R}^{(n+1)_{T}}, \max \left\{\operatorname { m a x } _ { k : 1 . . n } \left\{\underline{R}^{(k-1)_{T}} \circ_{T}\right.\right.\right. \\
& \left.\left.\left.\bar{R} \circ_{T} \underline{R}^{(n-k)_{T}}\right\} \circ_{T} \underline{R}, \underline{R}^{(n)_{T} \circ_{T}}\right\}\right] \\
& =\left[\underline{R}^{(n+1)_{T}}, \max _{k: 1 . . n+1}\left\{\underline{R}^{(k-1)_{T}} \circ_{T} \bar{R} \circ_{T}\right.\right. \\
& \left.\left.\quad \underline{R}^{(n-k)_{T}}\right\}\right]
\end{aligned}
$$

Theorem 4.2 Let $X$ be an arbitrary universe and $\mathscr{T}$ a sup-preserving $t$-norm. The transitive closure of $R$ is:

$$
R^{\mathscr{T}}={ }_{L} \operatorname{Sup}_{L \forall k \in \mathbb{N}}\left\{R^{(k) \mathscr{T}}\right\}
$$

Proof. Let $S=\operatorname{Sup}_{L \forall k \in \mathbb{N}}\left\{R^{(k) \mathscr{T}}\right\}$ be. By proposition 4.2:

$$
\begin{gathered}
S^{(2)_{\mathscr{T}}}={ }_{L}\left(\operatorname{Sup}_{L \forall k \in \mathbb{N}}\left\{R^{(k) \mathscr{T}}\right\}\right) \diamond_{\operatorname{Sup}_{L} \mathscr{T}} \\
\left(\operatorname{Sup}_{L \forall l \in \mathbb{N}}\left\{R^{(l) \mathscr{T}}\right\}\right)={ }_{L} \operatorname{Sup}_{L \forall k, l \in \mathbb{N}}\left\{R^{(k+l) \mathscr{T}}\right\}={ }_{L} \\
\operatorname{Sup}_{L \forall m \in \mathbb{N} \backslash\{0,1\}}\left\{R^{(m) \mathscr{T}}\right\} \subseteq S
\end{gathered}
$$

By lemma 4.1, it follows that $S$ is T-transitive.

Moreover, if $R \subseteq S^{\prime}$ and $S^{\prime}$ is T-transitive, then again by lemma 4.1 and 4.2 it holds that: 


$$
R^{(2)_{\mathscr{T}}} \subseteq_{L} S^{\prime(2)_{\mathscr{T}}} \subseteq_{L} S^{\prime}, \ldots, R^{(k) \mathscr{T}} \subseteq_{L} S^{\prime(k)_{\mathscr{T}}} \subseteq_{L} S^{\prime}
$$

hence $S \subseteq_{L} S^{\prime}$ and $R^{\mathscr{T}}={ }_{L} S$

Corollary 4.1 If $\mathscr{T}$ is t-representable with $T_{1}, T_{2}$ in $([0,1], \leqslant)$, the transitive closure of $R$ is:

$$
R^{\mathscr{T}}={ }_{L} \operatorname{Sup}_{L \forall k \in \mathbb{N}}\left\{\left[\underline{R}^{(k) T_{1}}, \bar{R}^{(k) T_{2}}\right]\right\}
$$

Corollary 4.2 If $\mathscr{T}$ is pseudo-t-representable with $T$, the transitive closure of $R$ is:

$$
R^{\mathscr{T}}={ }_{L} \operatorname{Sup} p_{L \forall k \in \mathbb{N}}\left\{\left[\underline{R}^{(n)_{T}}, \max _{q: 1 . . k}\left\{\underline{R}^{(q-1)_{T}} \circ_{T} \bar{R} \circ_{T}\right.\right.\right.
$$

Due associativity of $\mathscr{T}$ :

$$
\begin{gathered}
\mathscr{T}\left(\left[x_{1}, x_{2}\right], \mathscr{T}\left(\left[y_{1}, y_{2}\right],\left[z_{1}, z_{2}\right]\right)\right)={ }_{L} \\
\mathscr{T}\left(\mathscr{T}\left(\left[x_{1}, x_{2}\right],\left[y_{1}, y_{2}\right]\right),\left[z_{1}, z_{2}\right]\right)={ }_{L}\left[x_{1}, x_{2}\right] \Delta \\
{\left[y_{1}, y_{2}\right] \triangle\left[z_{1}, z_{2}\right]} \\
\operatorname{Inf}_{L}\left(\left[x_{1}, x_{2}\right], \operatorname{Inf}_{L}\left(\left[y_{1}, y_{2}\right],\left[z_{1}, z_{2}\right]\right)\right)={ }_{L} \\
\left.\operatorname{Inf}_{L}\left(\operatorname{Inf}_{L}\left(\left[x_{1}, x_{2}\right],\left[y_{1}, y_{2}\right]\right),\left[z_{1}, z_{2}\right]\right)\right)={ }_{L} \\
{\left[x_{1}, x_{2}\right] \triangle_{L-\text { inf }}\left[y_{1}, y_{2}\right] \triangle_{L-\text { inf }}\left[z_{1}, z_{2}\right]}
\end{gathered}
$$

Lemma 4.7 Let $\mathscr{T}$ be an arbitrary $t$-norm on L. Let $P$ be a path with a cycle:

$$
\begin{gathered}
P \equiv R\left(a, a_{1}\right) \triangle R\left(a_{1}, a_{2}\right) \Delta \ldots \Delta R\left(a_{t-1}, a_{t}\right) \triangle \\
R\left(a_{t}, c_{1}\right) \triangle R\left(c_{1}, c_{2}\right) \triangle \ldots \Delta R\left(c_{q}, a_{t}\right) \triangle \\
R\left(a_{t}, a_{t+1}\right) \triangle \ldots \Delta R\left(a_{k}, b\right)
\end{gathered}
$$

then

$$
P \equiv R\left(a, a_{1}\right) \triangle R\left(a_{1}, a_{2}\right) \triangle \ldots \triangle R\left(a_{t-1}, a_{t}\right) \triangle
$$
$R\left(a_{t}, c_{1}\right) \triangle R\left(c_{1}, c_{2}\right) \triangle \ldots \Delta R\left(c_{q}, a_{t}\right) \triangle R\left(a_{t}, a_{t+1}\right) \triangle$$$
\ldots \triangle R\left(a_{k}, b\right) \leqslant{ }_{L} R\left(a, a_{1}\right) \triangle R\left(a_{1}, a_{2}\right) \triangle \ldots \Delta
$$$$
R\left(a_{t-1}, a_{t}\right) \triangle R\left(a_{t}, a_{t+1}\right) \triangle \ldots \triangle R\left(a_{k}, b\right)
$$

Proof. Trivial due to $\mathscr{T}\left(\left[x_{1}, x_{2}\right],\left[y_{1}, y_{2}\right]\right) \leqslant{ }_{L}\left[y_{1}, y_{2}\right]$ for all $\left[x_{1}, x_{2}\right],\left[y_{1}, y_{2}\right]$ in $L$ and associativity of $\mathscr{T} \square$

Theorem 4.3 Let $X$ be a finite universe with cardinality $n$. The transitive closure of $R, R^{\mathscr{T}}$, is:

$$
R^{\mathscr{T}}={ }_{L} \operatorname{Sup}_{L k=1 . . n}\left\{R^{(k) \mathscr{T}}\right\}
$$

\section{Proof.}

$$
\begin{aligned}
& R^{(k)_{T}}(a, b)={ }_{L} \\
& \quad{ }_{L} \operatorname{Sup}_{L a_{1}, a_{2}, \ldots, a_{k-1}} R\left(a, a_{1}\right) \triangle R\left(a_{1}, a_{2}\right) \triangle \\
& \quad \ldots \triangle R\left(a_{k-1}, b\right) \\
& \quad \leqslant L \quad \operatorname{Sup}_{L a_{1}, a_{2}, \ldots, a_{k-1}} R\left(a, a_{1}\right) \quad \triangle_{I n f_{L}} \\
& \quad R\left(a_{1}, a_{2}\right) \triangle_{I n f_{L}} \ldots \triangle_{\operatorname{Inf}} R\left(a_{k-1}, b\right) \text { ac- } \\
& \quad \text { cording to lemma } 4.3 .
\end{aligned}
$$

Now suppose $k>n$. Then $R^{(k)} \subseteq_{L} R^{(n)}$ because it exists a cycle (lemma 4.7). Therefore, the cases $k>n$ need not be considered
Corollary 4.3 If $\mathscr{T}$ is $t$-representable with $T_{1}, T_{2}$ in $([0,1], \leqslant)$, the transitive closure of $R$ is:

$$
R^{\mathscr{T}}={ }_{L} \operatorname{Sup}_{L k=1 . . n}\left\{\left[\underline{R}^{(k)_{T_{1}}}, \bar{R}^{(k)_{T_{2}}}\right\}\right.
$$

Corollary 4.4 If $\mathscr{T}$ is pseudo-t-representable with $T$, the transitive closure of $R$ is:

$R^{\mathscr{T}}=_{L} \operatorname{Sup}_{L k=1 . . n}\left\{\left[\underline{R}^{(n)_{T}}, \max _{q: 1 . . k}\left\{\underline{R}^{(q-1)_{T}} \circ_{T} \bar{R} \circ_{T}\right.\right.\right.$

$$
\left.\left.\left.\underline{R}^{(k-q)_{T}}\right\}\right]\right\}
$$

Theorem 4.4 Let $X$ be a finite universe with cardinality $n$. If $R$ is a locally reflexive relation, the transitive closure of $R$ is:

$$
R^{\mathscr{T}}={ }_{L} \operatorname{Sup}_{L k=1 . . n-1}\left\{R^{(k) \mathscr{T}}\right\}
$$

The proof is a straightforward generalization of the proof for fuzzy relations.

Proof. If $R$ is a locally reflexive relation then $R(a, b) \leqslant{ }_{L} R(a, a)$. Consider a cycle of length $n$, then:

$$
\begin{aligned}
& R^{(n)} \mathscr{T}(a, a)=_{L} \\
& \quad{ }_{L} \operatorname{Sup}_{L a_{1}, a_{2}, \ldots, a_{k-1}} R\left(a, a_{1}\right) \triangle R\left(a_{1}, a_{2}\right) \triangle \\
& \quad \ldots, \triangle R\left(a_{k-1}, a\right) \leqslant L \\
& \quad \operatorname{Sup}_{L a_{1}, a_{2}, \ldots, a_{k-1}} R\left(a, a_{1}\right) \triangle_{I n f_{L}} R\left(a_{1}, a_{2}\right) \triangle_{I n f_{L}} \\
& \quad \ldots \triangle_{I n f_{L}} R\left(a_{k-1}, a\right){ }_{L} R(a, a)
\end{aligned}
$$

Theorem 4.5 Let $X$ be a finite universe with cardinality $n$. If $R$ is a reflexive fuzzy relation on $X$ then the transitive closure of $R$ is:

$$
R^{\mathscr{T}}={ }_{L} R^{(n-1)_{\mathscr{T}}}
$$

Proof. The proof is a straightforward generalization of the proof for fuzzy relations.

$$
\begin{aligned}
R(a, b)^{(k)_{\mathscr{T}}}={ }_{L} & \\
& ={ }_{L} \mathscr{T}\left(R(a, a), R^{(k) \mathscr{T}}(a, b)\right) \\
& \leqslant{ }_{L} \operatorname{Sup}_{L c \in X}\left\{T\left(R(a, c), R^{(k)_{\mathscr{T}}}(c, b)\right)\right\} \\
& ={ }_{L} R^{(k+1)_{\mathscr{T}}}(a, b)
\end{aligned}
$$

Therefore $R(a, b)^{(k)_{\mathscr{T}}} \leqslant_{L} R^{(k+1)_{\mathscr{T}}}(a, b) \Leftrightarrow R^{(k)_{\mathscr{T}}} \subseteq$ $R^{(k+1)_{\mathscr{T}}}$

Corollary 4.5 If $\mathscr{T}$ is $t$-representable with $T_{1}, T_{2}$ in $([0,1], \leqslant)$, the transitive closure of $R$ is:

$$
R^{\mathscr{T}}={ }_{L}\left\{\left[\underline{R}^{(n-1)_{T_{1}}}, \bar{R}^{(n-1)_{T_{2}}}\right]\right\}
$$

Corollary 4.6 If $\mathscr{T}$ is pseudo-t-representable with $T$, the transitive closure of $R$ is:

$$
\begin{gathered}
R^{\mathscr{T}}=_{L} \\
\left\{\left[\underline{R}^{(n-1)_{T}}, \max _{q: 1 . . n-1}\left\{\underline{R}^{(q-1)_{T}} \circ_{T} \bar{R} \circ_{T} \underline{R}^{(n-1-q)_{T}}\right\}\right]\right\}
\end{gathered}
$$


Definition 4.5 A distance $d$ of the supremum on $L$ is defined for all $x_{1}, x_{2}, y_{1}, y_{2} \in[0,1]$ by $d\left(\left[x_{1}, x_{2}\right],\left[y_{1}, y_{2}\right]\right)=\max \left(\left|x_{1}-y_{1}\right|,\left|x_{2}-y_{2}\right|\right)$.

Definition 4.6 Let $R, S$ be two interval-valued fuzzy relations on a set $X$. The distance $d$ between $R$ and $S$ is defined by:

$$
d(R, S)=\sup _{x, y \in X} d(R(x, y), S(x, y))
$$

Lemma $4.8 d$ is a distance on the set $R_{X}$ of intervalvalued fuzzy relations on $X$.

\section{Proof.}

The supremum of distances is a distance.

Theorem 4.6 Let $T$ be a generalized continuous $t$ norm and $R_{X}$ the set of interval-valued fuzzy relations on $X . R_{X}$ with the sup- $\mathscr{T}$ product is an ordered topological semigroup.

\section{Proof.}

- Associativity is a straightforward exercise.

- The interval-valued relation $E(x, y)=$ $\left\{\begin{array}{ll}{[1,1],} & x=y ; \\ {[0,0],} & \text { otherwise. }\end{array}\right.$ is the identity element of $R_{X}$.

- Continuity: Since $T$ is defined on a compact set, it is uniformly continuous. Therefore:

$$
\begin{gathered}
\forall \varepsilon>0, \exists \delta>0 \text { such that } \\
\forall m, n, m^{\prime}, n^{\prime}, a, b, a^{\prime}, b^{\prime} \in[0,1] \\
\max \left(|m-a|,\left|m^{\prime}-a^{\prime}\right|,|n-b|,\left|n^{\prime}-b^{\prime}\right|\right) \Rightarrow \\
\mid \mathscr{T}\left(\left[m, m^{\prime}\right],\left[n, n^{\prime}\right]\right)-\mathscr{T}\left(\left[a, a^{\prime}\right],\left[b, b^{\prime}\right]\right)<\varepsilon\left(^{*}\right)
\end{gathered}
$$

We want to prove that given two interval-valued fuzzy relations $A, B \in R_{X}$ :

$$
\forall \varepsilon>0, \exists \delta>0 \text { such that } \forall M, N \in R_{X}
$$

$\max (d(M, A), d(N, B))<\delta \Rightarrow d(M \circ N, A \circ B)<\varepsilon$

Given $\varepsilon$, we take $\delta>0$ satisfying (*). Then:

$$
\begin{gathered}
d(M \circ N, A \circ B)= \\
\sup _{x, y \in X} \mid \sup _{z \in X} \mathscr{T}(M(x, z), N(z, y))- \\
\sup _{z \in X} \mathscr{T}(A(x, z), B(z, y)) \mid \leqslant \\
\sup _{x, y \in X} \sup _{z \in X} \mid \mathscr{T}(M(x, z), N(z, y))- \\
\mathscr{T}(A(x, z), B(z, y)) \mid \leqslant \varepsilon
\end{gathered}
$$

- Monotonicity is an immediate consequence of the monotonicity of $\mathscr{T}$

Corollary 4.7 For any positive integer $n$ the map assigning to an interval-valued fuzzy relation $M$ on a finite set its $n^{\text {th }}$ power is non-decreasing and continuous if the corresponding t-norm on $L \mathscr{T}$ is continuous.

Corollary 4.8 If a t-norm on $L \mathscr{T}$ is continuous, the map that assigns the $\mathscr{T}$-transitive closure to the interval-valued fuzzy relations on a finite set is nondecreasing and continuous.

It is given a method to calculate the transitive closure of a interval-valued fuzzy relation. Nevertheless, there are more effective methods and algorithms. For example, it is possible to use an extension of the Floyd-Warshall algorithm ${ }^{17}$

\section{Algorithm to compute the $\mathscr{T}$-transitive closure of an interval-valued fuzzy relation}

Let $R$ be an interval-valued relation on a finite universe $X$ with cardinality $n$ and let $\mathscr{T}$ be a t-norm on $L$. The $\mathscr{T}$-transitive closure of $R, R^{\mathscr{T}}$, can be computed using the following algorithm:

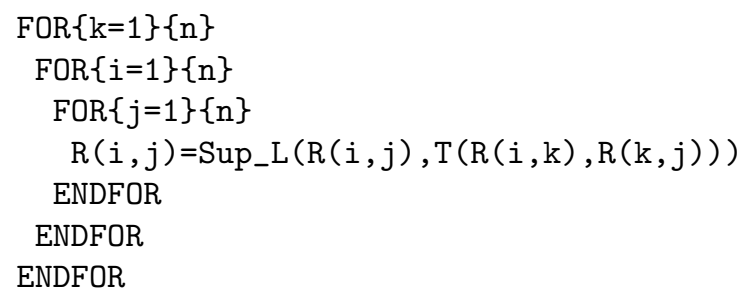

Example 5.1 Let $\mathscr{T}$ be a t-norm on $L$

$\operatorname{Inf}_{L}\left(\left\{\left[x_{1}, x_{2}\right],\left[y_{1}, y_{2}\right]\right\}\right)={ }_{L}\left[\min \left(x_{1}, y_{1}\right), \min \left(x_{2}, y_{2}\right)\right]$

and let $R: X \times X \rightarrow L$ be the following intervalvalued relation:

$$
R=\left(\begin{array}{cccc}
{[1,1]} & {[0.6,0.8]} & {[0.6,0.9]} & {[0.0]} \\
& {[1,1]} & {[0.4,0.9]} & {[0.6,0.9]} \\
& {[1,1]} & {[0.6,0.9]} \\
& & & {[1,1]}
\end{array}\right)
$$

The computation of the $\mathscr{T}$-transitive closure of $R$ is the following:

$$
R=\left(\begin{array}{cccc}
{[1,1]} & {[0.6,0.8]} & {[0.6,0.9]} & {[0,0]} \\
& {[1,1]} & {[0.6,0.9]} & {[0.6,0.9]} \\
& {[1,1]} & {[0.6,0.9]} \\
& & {[1,1]}
\end{array}\right)
$$




$$
\begin{array}{r}
R=\left(\begin{array}{cccc}
{[1,1]} & {[0.6,0.8]} & {[0.6,0.9]} & {[0.6,0.8]} \\
& {[1,1]} & {[0.6,0.9]} & {[0.6,0.9]} \\
& & {[1,1]} & {[0.6,0.9]} \\
& & & {[1,1]}
\end{array}\right) \\
R=\left(\begin{array}{cccc}
{[1,1]} & {[0.6,0.9]} & {[0.6,0.9]} & {[0.6,0.9]} \\
& {[1,1]} & {[0.6,0.9]} & {[0.6,0.9]} \\
& {[1,1]} & {[0.6,0.9]} \\
& & & {[1,1]}
\end{array}\right) \\
R^{\mathscr{T}}=\left(\begin{array}{cccc}
{[1,1]} & {[0.6,0.9]} & {[0.6,0.9]} & {[0.6,0.9]} \\
& {[1,1]} & {[0.6,0.9]} & {[0.6,0.9]} \\
& & {[1,1]} & {[0.6,0.9]} \\
& & & {[1,1]}
\end{array}\right)
\end{array}
$$

Note that $R^{\mathscr{T}}=\left[\underline{R}^{\mathrm{min}}, \bar{R}^{\mathrm{min}}\right]$ which we already by theorem 4.1.

Example 5.2 Let $\mathscr{T}$ be a t-norm on $L \quad T_{w}\left(\left[x_{1}, x_{2}\right],\left[y_{1}, y_{2}\right]\right)={ }_{L} \quad\left[\max \left(0, x_{1}+y_{1}-\right.\right.$ $\left.1), \max \left(0, x_{2}+y_{2}-1\right)\right]$ and let $R: X \times X \rightarrow L$ be the following interval-valued relation:

$$
R=\left(\begin{array}{cccc}
{[1,1]} & {[0.6,0.8]} & {[0.6,0.9]} & {[0,0]} \\
& {[1,1]} & {[0.4,0.9]} & {[0.6,0.9]} \\
& & {[1,1]} & {[0.6,0.9]} \\
& & & {[1,1]}
\end{array}\right)
$$

The computation of the $\mathscr{T}$-transitive closure of $R$ is the following:

$$
\begin{aligned}
& R=\left(\begin{array}{cccc}
{[1,1]} & {[0.6,0.8]} & {[0.6,0.9]} & {[0,0]} \\
& {[1,1]} & {[0.4,0.9]} & {[0.6,0.9]} \\
& & {[1,1]} & {[0.6,0.9]} \\
& & & {[1,1]}
\end{array}\right) \\
& R=\left(\begin{array}{cccc}
{[1,1]} & {[0.6,0.8]} & {[0.6,0.9]} & {[0.2,0.7]} \\
& {[1,1]} & {[0.4,0.9]} & {[0.6,0.9]} \\
& & {[1,1]} & {[0.6,0.9]} \\
& & & {[1,1]}
\end{array}\right) \\
& R=\left(\begin{array}{cccc}
{[1,1]} & {[0.6,0.8]} & {[0.6,0.9]} & {[0.2,0.8]} \\
& {[1,1]} & {[0.4,0.9]} & {[0.6,0.9]} \\
& & {[1,1]} & {[0.6,0.9]} \\
& & & {[1,1]}
\end{array}\right) \\
& R^{\mathscr{T}}=\left(\begin{array}{cccc}
{[1,1]} & {[0.6,0.8]} & {[0.6,0.9]} & {[0.2,0.8]} \\
& {[1,1]} & {[0.4,0.9]} & {[0.6,0.9]} \\
& & {[1,1]} & {[0.6,0.9]} \\
& & & {[1,1]}
\end{array}\right)
\end{aligned}
$$

Note that $R^{\mathscr{T}}=\left[\underline{R}^{W}, \bar{R}^{W}\right]$ also known by theorem 4.1
Example 5.3 Let $\mathscr{T}$ be a t-norm on $L$ $T\left(\left[x_{1}, x_{2}\right],\left[y_{1}, y_{2}\right]\right)=_{L}\left[W\left(x_{1}, y_{1}\right), \min \left(x_{2}, y_{2}\right)\right]$ and let $R: X \times X \rightarrow L$ be the following interval-valued relation:

$$
R=\left(\begin{array}{cccc}
{[1,1]} & {[0.6,0.8]} & {[0.6,0.9]} & {[0,0]} \\
& {[1,1]} & {[0.4,0.9]} & {[0.6,0.9]} \\
& & {[1,1]} & {[0.6,0.9]} \\
& & & {[1,1]}
\end{array}\right)
$$

The computation of the $\mathscr{T}$-transitive closure of $R$ is the following:

$$
\begin{aligned}
& R=\left(\begin{array}{cccc}
{[1,1]} & {[0.6,0.8]} & {[0.6,0.9]} & {[0,0]} \\
& {[1,1]} & {[0.4,0.9]} & {[0.6,0.9]} \\
& {[1,1]} & {[0.6,0.9]} \\
& & & {[1,1]}
\end{array}\right) \\
& R=\left(\begin{array}{cccc}
{[1,1]} & {[0.6,0.8]} & {[0.6,0.9]} & {[0.2,0.8]} \\
& {[1,1]} & {[0.4,0.9]} & {[0.6,0.9]} \\
& & {[1,1]} & {[0.6,0.9]} \\
& & & {[1,1]}
\end{array}\right) \\
& R=\left(\begin{array}{cccc}
{[1,1]} & {[0.6,0.8]} & {[0.6,0.9]} & {[0.2,0.9]} \\
& {[1,1]} & {[0.4,0.9]} & {[0.6,0.9]} \\
& {[1,1]} & {[0.6,0.9]} \\
& & & {[1,1]}
\end{array}\right) \\
& R^{\mathscr{T}}=\left(\begin{array}{cccc}
{[1,1]} & {[0.6,0.8]} & {[0.6,0.9]} & {[0.2,0.9]} \\
& {[1,1]} & {[0.4,0.9]} & {[0.6,0.9]} \\
& & {[1,1]} & {[0.6,0.9]} \\
& & & {[1,1]}
\end{array}\right)
\end{aligned}
$$

Note that $R^{\mathscr{T}}=\left[\underline{R}^{W}, \bar{R}^{\mathrm{min}}\right]$ also known by theorem 4.1.

Example 5.4 Let $\mathscr{T}$ be a t-norm on $L \quad T_{W}\left(\left[x_{1}, x_{2}\right],\left[y_{1}, y_{2}\right]\right)={ }_{L} \quad\left[\max \left(0, x_{1}+y_{1}-\right.\right.$ $\left.1), \max \left(0, x_{1}+y_{2}-1, x_{2}+y_{1}-1\right)\right]$ and let $R$ : $X \times X \rightarrow L$ be the following interval-valued relation:

$$
R=\left(\begin{array}{cccc}
{[1,1]} & {[0.6,0.8]} & {[0.6,0.9]} & {[0,0]} \\
& {[1,1]} & {[0.4,0.9]} & {[0.6,0.9]} \\
& {[1,1]} & {[0.6,0.9]} \\
& & & {[1,1]}
\end{array}\right)
$$

The computation of the $\mathscr{T}$-transitive closure of $R$ is the following:

$$
R=\left(\begin{array}{cccc}
{[1,1]} & {[0.6,0.8]} & {[0.6,0.9]} & {[0,0]} \\
& {[1,1]} & {[0.4,0.9]} & {[0.6,0.9]} \\
& & {[1,1]} & {[0.6,0.9]} \\
& & & {[1,1]}
\end{array}\right)
$$




$$
\begin{array}{r}
R=\left(\begin{array}{cccc}
{[1,1]} & {[0.6,0.8]} & {[0.6,0.9]} & {[0.2,0.5]} \\
& {[1,1]} & {[0.4,0.9]} & {[0.6,0.9]} \\
& & {[1,1]} & {[0.6,0.9]} \\
& & & {[1,1]}
\end{array}\right) \\
R=\left(\begin{array}{cccc}
{[1,1]} & {[0.6,0.8]} & {[0.6,0.9]} & {[0.2,0.5]} \\
& {[1,1]} & {[0.4,0.9]} & {[0.6,0.9]} \\
& {[1,1]} & {[0.6,0.9]} \\
& & {[1,1]}
\end{array}\right) \\
R^{\mathscr{T}}=\left(\begin{array}{cccc}
{[1,1]} & {[0.6,0.8]} & {[0.6,0.9]} & {[0.2,0.5]} \\
& {[1,1]} & {[0.4,0.9]} & {[0.6,0.9]} \\
& & {[1,1]} & {[0.6,0.9]} \\
& & & {[1,1]}
\end{array}\right)
\end{array}
$$

Note that the generalized $\mathscr{T}$-norm in this example is not t-representable.

\section{An Application}

It is possible to model preference relations using Interval-valued Fuzzy Sets. For example, it is possible to model the relation "be better or equal than". Let $R$ be a relation which describes the degree of preference between pairs of objects. This relation is reflexive and transitive. In this context, the $\mathscr{T}$ transitive closure can be used to impose coherence to a set of dates.

Example 6.1 Let $R$ be an interval-valued relation which models the degree of the relation "be better or equal than" between four objects $\{a, b, c, d\}$. It is possible to model the transitivity of this relation using the $t$-norm on $L *_{L}\left(\left[x_{1}, x_{2}\right],\left[y_{1}, y_{2}\right]\right)={ }_{L}$ $\left[x_{1} * y_{1}, x_{2} * y_{2}\right]$ :

$$
R=\left(\begin{array}{cccc}
{[1,1]} & {[0.6,0.9]} & {[0.6,0.7]} & {[0,0]} \\
& {[1,1]} & {[0,0]} & {[0.2,0.8]} \\
& & {[1,1]} & {[0,0]} \\
& & & {[1,1]}
\end{array}\right)
$$

However, $R$ is not a $*_{L}$-transitive relation so some membership degrees must be wrong.

$$
\left(\begin{array}{cccc}
{[1,1]} & {[0.6,0.9]} & R^{\mathscr{T}}= & \\
& {[1,1]} & {[0.6,0.7]} & {[0.16,0.72]} \\
& & {[1,1]} & {[0.096,0.5]} \\
& & & {[1,1]}
\end{array}\right)
$$

It is possible to impose transitivity replacing $R$ by $R^{\mathscr{T}}$.

\section{Conclusions}

In this paper the main properties of interval-valued fuzzy relations are studied: reflexivity, symmetry, composition, local reflexivity and T-transitivity. The existence and uniqueness of the T-transitive closure for $\mathscr{L}$-fuzzy relations has been proved when $\mathscr{L}$ is a complete lattice. Several expressions to compute the T-transitive closure of $\mathscr{I} \mathscr{V} \mathscr{F} \mathscr{R}$ are proposed for any t-norm on $L$, any generalized $\mathrm{t}$ representable t-norm and any t-pseudo representable t-norm. Finally, an algorithm based on the FloydWarshall method to compute the T-transitive closure of $\mathscr{I} \mathscr{V} \mathscr{F} \mathscr{R}$ for any t-norm on $L \mathrm{~T}$ is showed. Some examples are provided.

\section{Acknowledgments}

This research is partially supported by the Spanish Ministry of Science and Technology, grant number TIN2009-07901, the Research Group CAM GR58/08 at Complutense University of Madrid.

\section{References}

1. K.T. Atanassov. Intuitionistic Fuzzy Sets. PhysicaVerlag, Heidelberg, New York, 1999.

2. B. De Baets and H. De Meyer. On the existence and construction of t-transitive closures. Inf. Sci., 152(1):167-179, 2003.

3. P. Burillo and H. Bustince. Intuitionistic fuzzy relations (part i). Mathware Soft Comput, 2:5-38, 1995.

4. P. Burillo and H. Bustince. Structures on intuitionistic fuzzy relations. Fuzzy Sets and Systems, 78:293-303, 1996.

5. H. Bustince, F. Herrera, and J. Montero. Fuzzy Sets and Their Extensions: Representation, Aggregation and Models, volume 220 of Studies in Fuzziness and Soft Computing. Hardcover, 2008.

6. C. Cornelis, G. Deschrijver, and E. Kerre. Advances and challenges in interval-valued fuzzy logic. Fuzzy Sets and Systems, 157(5):622-627, 2006.

7. C. Cornelis, G.Deschrijver, and E. Kerre. Implication in intuitionistic fuzzy and interval-valued fuzzy set theory: construction, classification, application. Int. J. Approx. Reasoning, 35(1):55-95, 2004.

8. Bernard De Baets and Radko Mesiar. Triangular norms on product lattices. Fuzzy Sets Syst., 104:6175, May 1999. 
9. G. Deschrijver and E. E. Kerre. On the cuts of intuitionistic fuzzy compositions. Kuwait Journal of Science and Engineering, 32(1):17-38, 2005.

10. Glad Deschrijver. Arithmetic operators in intervalvalued fuzzy set theory. Inf. Sci., 177(14):2906-2924, 2007.

11. Glad Deschrijver and Etienne E. Kerre. On the composition of intuitionistic fuzzy relations. Fuzzy Sets Syst., 136(3):333-361, 2003.

12. Urszula Dudziak and Barbara Pȩkala. Intuitionistic fuzzy preference relations. In Atlantis Press, editor, EUSFLAT-LFA, pages 529-536, 2011.

13. J. Goguen. L-fuzzy sets. Journal Of Mathematical Analysis And Applications, 18:145-174, 1967.

14. I. Grattan-Guiness. Fuzzy membership mapped onto interval and many-valued quantities. Math. Logik. Grundladen Math, 22:149-160, 1975.

15. D. Gomez J. Montero and Bustince H. On the relevance of some families of fuzzy sets. Fuzzy Sets And Systems, 158:2429-2442, 2007.

16. K.U. Jahn. Intervall-wertige Mengen. Math. Nach., 68:115-132, 1975.

17. H. Naessens, H. De Meyer, and De B. Baets. Algorithms for the computation of $\mathrm{T}$-transitive closures.
IEEE Transactions on Fuzzy Systems, 10(1):541-551, 2002.

18. Barbara Pekala. Properties of interval-valued fuzzy relations, atanassov's operators and decomposable operations. In Eyke Hllermeier, Rudolf Kruse, and Frank Hoffmann, editors, IPMU (1), volume 80 of Communications in Computer and Information Science, pages 647-655. Springer, 2010.

19. E. Sanchez and R. Sambuc. Fuzzy relationships. phi -fuzzy functions. application to diagnostic aid in thyroid pathology. Proceedings of an International Symposium on Medical Data Processing, pages 513-524, 1976.

20. L.A. Zadeh. Fuzzy sets. Information And Control, 8:338-353, 1965.

21. L.A. Zadeh. The concept of a linguistic variable and its application to approximate reasoning i. Information Sciences, 8:199-249, 1975.

22. L.A. Zadeh. The concept of a linguistic variable and its application to approximate reasoning ii. Information Sciences, 8:301-357, 1975.

23. L.A. Zadeh. The concept of a linguistic variable and its application to approximate reasoning iii. Information Sciences, 9:43-80, 1975. 